\author{
Л. И. Алексеева ${ }^{*}{ }^{* *}, \mathbf{1}$, доктор медицинских наук, профессор \\ Н. Г. Кашеварова*, кандидат медицинских наук \\ * ФГБНУ НИИР им. В. А. Насоновой, Москва, Россия \\ ** ФГБОУ ДПО РМАНПО Минздрава России, Москва, Россия
}

Резюме. Наличие болевого синдрома и нарастание функциональной недостаточности суставов - наиболее частые причины обращения пациентов за медицинской помощью. В настоящее время, учитывая рост распространенности остеоартрита, необходим более активный мультимодальный подход к ведению пациентов с учетом их индивидуальных особенностей и потребностей. Основными целями терапии остеоартрита являются: уменьшение боли, сохранение или улучшение функции суставов, предотвращение нарастания функциональной недостаточности, улучшение качества жизни, связанного со здоровьем, и уменьшение/предотвращение структурного прогрессирования заболевания. В статье представлены данные об одном из эффективных и безопасных методов лечения остеоартрита коленных и тазобедренных суставов - внутрисуставном введении гиалуроновой кислоты, который обеспечивает значительное снижение боли, улучшение функции сустава, способствует снижению потребности в нестероидных противовоспалительных препаратах и замедлению прогрессирования заболевания. Полученные результаты исследований свидетельствуют о хорошем и длительном симптоматическом эффекте препарата, высоком профиле безопасности и возможности его использования в широкой клинической практике.

Ключевые слова: остеоартрит, коленные суставы, тазобедренные суставы, гиалуроновая кислота, внутрисуставные инъекции. Для цитирования: Алексеева Л. И., Кашеварова Н. Г. Эффективность внутрисуставных инъекций высокомолекулярной гиалуроновой кислоты при остеортрите коленных и тазобедренных суставов // Лечащий Врач. 2021; 7 (24): 39-44. DOI: 10.51793/ OS.2021.24.7.008

\title{
Efficacy of intra-articular of high molecular weight hyaluronic acid injections in in osteoarthritis of the knee and hip
}

\author{
L. I. Alekseeva*, ${ }^{* *}, 1$, N. G. Kashevarova* \\ ${ }^{*}$ V. A. Nasonova Research Institute of Rheumatology, Moscow, Russia \\ ** Department of Rheumatology, Russian Medical Academy of Continuing Professional Education, Ministry of Health of Russia, Moscow, \\ Russia
}

Abstract. The presence of pain syndrome and an increase in functional insufficiency of the joints are the most frequent reasons for patients seeking medical help. Currently, given the growing prevalence of osteoarthritis, a more active multimodal approach to patient management is needed, taking into account their individual characteristics and needs. The main goals of osteoarthritis therapy are: pain relief; maintaining or improving joint function; preventing the build-up of functional impairment, improving health-related quality of life, and reducing/preventing structural progression of the disease. The article presents data on one of the effective and safe methods of treating osteoarthritis of the knee and hip joints - intra-articular administration of hyaluronic acid, which provides a significant reduction in pain, improvement of joint function; helps to reduce the need for NSAIDs and slow the progression of the disease. The obtained research results indicate a good and long-term symptomatic effect of the drug, a high safety profile and the possibility of its use in wide clinical practice.

Keywords: osteoarthritis, knee joints, hip joints, hyaluronic acid, intra-articular injections.

For citation: Alekseeva L. I., Kashevarova N. G. Efficacy of intra-articular of high molecular weight hyaluronic acid injections in in osteoarthritis of the knee and hip // Lechaschy Vrach. 2021; 7 (24): 39-44. DOI: 10.51793/OS.2021.24.7.008

\footnotetext{
${ }^{1}$ Контактная информация: dr.alekseeva@gmail.com
} 


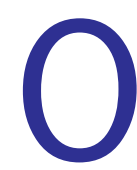

стеоартрит (ОА) - самое частое заболевание суставов, в основе которого лежит поражение всех компонентов сустава, в первую очередь хряща, а также субхондральной кости, синовиальной оболочки, связок, капсулы, околосуставных мышц, приводящее к развитию болевого синдрома, снижению функциональной активности и качества жизни пациента. Наиболее часто поражаются нагрузочные суставы (коленные, тазобедренные и суставы кистей). Распространенность ОА различна в зависимости от изучаемой популяции и применяемого эпидемиологического метода. Так, заболеваемость ОА коленных суставов (ОАК) при использовании для диагностики как клинических и рентгенологических критериев, так и их комбинации составляет от $1,5 \%$ до 42,4\%. Распространенность ОАК нарастает с увеличением возраста больных, причем она выше у лиц женского пола. Распространенность ОА суставов кистей варьирует от 2,0\% до $16,3 \%$, это заболевание также чаще выявляется у женщин и имеет возрастную зависимость. Поражение тазобедренных суставов диагностируют значительно реже, чем ОАК и суставов кистей. Причем многие исследователи не находят значимых гендерных различий при ОА данной локализации [1].

Согласно исследованию Global Burden of Disease 2010 г., из 291 нозологии ОАК и тазобедренных суставов занимает 11-е место по количеству случаев инвалидизации пациентов в мире и 38-е - по числу лет, прожитых с инвалидностью. Распространенность ОАК была 3,8\% (95\% ДИ 3,6-4,1\%), а тазобедренных - 0,85\% (95\% ДИ 0,74-1,02\%) [2].

Крупное популяционное когортное исследование $(\mathrm{n}=1163)$ продемонстрировало, что пациенты с ОА тазобедренных суставов имеют более высокий риск смертности по сравнению с популяцией в целом $(1,55,95 \%$ ДИ 1,41-1,70). Авторы выделили основные предикторы смертности у данных пациентов: мужской пол $(1,59,95 \%$ ДИ 1,30-1,96), сахарный диабет $(1,95$, 95\% ДИ 1,31-2,90), сердечно-сосудистые заболевания $(1,38$, $95 \%$ ДИ 1,12-1,71), наличие онкологической патологии $(2,28$, 95\% ДИ 1,50-3,47), о которых сообщалось на исходном уровне, и выраженное нарушение функции суставов, препятствующее нормальному движению $(1,48,95 \%$ ДИ 1,17-1,86). Причем при более выраженной функциональной недостаточности (ФН) риск смертности был выше $(\mathrm{p}<0,001)$ [3].

Наличие болевого синдрома и нарастание ФН суставов наиболее частые причины обращения пациентов за медицинской помощью. В настоящее время, учитывая рост распространенности ОА, необходим более активный мультимодальный подход к ведению пациентов с учетом их индивидуальных особенностей и потребностей.

Основными целями терапии ОА являются:

- уменьшение боли;

- сохранение или улучшение функции суставов;

- предотвращение нарастания ФН;

- улучшение качества жизни, связанного со здоровьем;

- уменьшение/предотвращение структурного прогрессирования заболевания.

Данная терапия включает комбинацию нефармакологических и фармакологических методов лечения и персонифицированно назначается в зависимости от степени поражения суставов.

За последнее время ведущие международные и российские сообщества - Международное общество по изучению остеоартрита (Osteoarthritis Research Society International, OARSI, 2019 г.) [4], Европейское общество по клиническим и экономическим аспектам остеопороза, остеоартрита и мышечно-скелетных заболеваний (European Society for Clinical and Economic
Aspects of Osteoporosis, Osteoarthritis and Musculoskeletal Diseases, ESCEO, 2019 г.) [5], Американская коллегия ревматологии (American College of Rheumatology, ACR, 2019 г.) [6], Европейская антиревматическая лига (European League Against Rheumatism, EULAR, 2018 г.) [7], Ассоциация ревматологов России (АPP, 2016 г.) [8] и др. предлагают обновленные рекомендации по ведению больных с ОА. Комплексный план лечения у отдельного пациента должен включать образовательные, поведенческие, психосоциальные и физические вмешательства, а также использование локальных, пероральных и внутрисуставных препаратов. Рекомендации ACR по лечению ОА суставов кистей, коленных и тазобедренных суставов предлагают использовать различные варианты немедикаментозных и фармакологических методов терапии через различные временные промежутки в зависимости от характера течения заболевания пациента [6], поскольку одни больные могут достигать адекватного ответа на терапию после назначения одного ее метода, в то время как другим для достижения оптимальных результатов может потребоваться последовательное или комбинированное применение различных способов лечения.

Рекомендации OARSI 2019 г. подчеркивают важность немедикаментозных методов, включающих обучение пациентов, снижение веса (при избыточной массе тела), физические упражнения и уменьшение нагрузки на коленные суставы при ОА данной локализации [4]. В описательном обзоре представлены все возможные факторы риска ОА, часть из которых поддается модификации. Авторы утверждают, что целостный подход к лечению ОАК может состоять из упражнений по укреплению четырехглавой мышцы бедра, дополненных правильной диетой, направленной на снижение веса, профессиональной реабилитацией, лечением сопутствующих заболеваний и ортопедической коррекцией [9]. Программа модификации индивидуальных факторов риска должна быть разработана в соответствии с предпочтениями и привычками пациента, местом работы, историей болезни и общим состоянием здоровья.

Пошаговая медикаментозная терапия ОА прекрасно представлена в рекомендациях ESCEO, 2019 г., а первый ее шаг назначение симптоматических препаратов замедленного действия (SYSADOA, Symptomatic Slow Activity Drugs for Osteoarthritis), которые определены как «базисные» средства лечения ОА. Препараты данной группы обладают не только анальгетическим и противовоспалительным эффектами, но и болезнь-модифицирующим действием, иными словами, способны замедлять прогрессирование заболевания.

Помимо пероральных SYSADOA к этой группе относятся и внутрисуставные (в/с) лекарственные средства, например, препараты гиалуроновой кислоты (ГиК), которые широко и успешно используются в реальной клинической практике. Их назначают при неэффективности предшествующей терапии, при наличии противопоказаний к приему нестероидных противовоспалительных препаратов (НПВП) и др.

ГиК была открыта К. Майером (К. Meyer) и Дж. Пальмером (John W. Palmer) в 1934 г. [10] и в настоящее время продолжает привлекать пристальное внимание химиков, биохимиков, биоинженеров и других исследователей из различных научных областей. Она является важным компонентом экстрацеллюлярного матрикса, обнаруживается и внутри клеток [11]. Содержание ГиК в тканях различно: например, гребни петуха содержат 7,50 мг/мл, пуповина плода - 4,10 мг/мл, синовиальная жидкость суставов человека - 1,50-3,60 мг/мл, стекловидное тело - 0,14-0,34 мг/г, дерма и эпидермис - 0,20-0,50 и 0,10 мг/г соответственно [12]. ГиК может быть животного 
происхождения, а также полученная на основе бактерий, например, рода Streptococcus (uberis, equisimilis, zooepidermicus, pyogenes, equi), Pasteurella multocida и Corynebacterium glutamicum; зеленых водорослей Chlorella; сахаромицет (Cryptococcus neoformans); моллюсков (двустворчатый моллюск Mytilus galloprovincialis). В то же время ГиК не обнаружена у грибов, насекомых или растений [13-16].

В зависимости от источника своего происхождения ГиК имеет различную молекулярную массу. Выше всего она у ГиК, полученной из животных материалов. Например, петушиные гребни содержат ГиК массой 1200 кДа, пупочные канатики 3400 кДа, стекловидное тело крупного рогатого скота 770-1700 кДа. Напротив, бактериальная ГиК имеет молекулярную массу от 1000 до 4000 кДа, однако ферментативный метод позволяет получать полисахариды с диапазоном молекулярной массы от 550 кДа до 2500 кДа. Молекулярная масса ГиК зависит также от некоторых других условий: например, в синовиальной жидкости здорового человека она равна 6000-7000 кДа, а при ревматоидном артрите - меньше или равна $3000-5000$ кДа [17].

Наличие ГиК во многих тканях определяет ее широкое использование в медицине в качестве протеза синовиальной жидкости при лечении заболеваний суставов; в косметологии и эстетической медицине - в качестве кожных наполнителей и в офтальмологии - в качестве искусственной слезы. Внутрисуставная терапия - локальный метод лечения, позволяющий избежать нежелательных явлений (НЯ), часто наблюдаемых при применении системных препаратов, в частности НПВП [18].

В настоящее время во многих клинических исследованиях и метаанализах продемонстрировано существенное превосходство препаратов ГиК над плацебо и НПВП при ОАК. Так, R. R. Bannuru и соавт. в проведенном метаанализе при участии 606 пациентов с ОА показали, что в/с введение ГиК было эффективно в плане уменьшения боли через 4 недели от начала лечения $(\mathrm{ES}=0,31 ; 95 \%$ ДИ 0,17-0,45), а максимальная эффективность достигалась через 8 недель $(\mathrm{ES}=0,46$; 95\% ДИ 0,28-0,65), и эффект сохранялся до 24 недель ( $\mathrm{ES}=0,21$; 95\% ДИ 0,10-0,31). И хотя препараты ГиК характеризуются медленным началом действия, их максимальная эффективность была выше, чем у анальгетиков, например, парацетамола $(\mathrm{ES}=0,13 ; 95 \%$ ДИ 0,04-0,22), неселективных НПВП (ES = 0,$29 ; 95 \%$ ДИ 0,22-0,35) и ингибиторов ЦОГ-2 (ES = 0,44; 95\% ДИ 0,33-0,55) [19]. В другом метаанализе продемонстрировано преимущество ГиК над плацебо в течение 3 месяцев наблюдения по анальгетическому действию $(\mathrm{ES}=0,34$; 95\% ДИ 0,26-0,42) [20]. На основании системного обзора 137 исследований (33 243 больных ОА) было установлено, что ГиК не только значимо снижает боль и улучшает функцию сустава, но и снижает потребность в обезболивающих препаратах, замедляет прогрессирование заболевания и отодвигает необходимость в оперативном лечении [21]. Существуют немногочисленные данные о влиянии ГиК на течение ОА. Так, ретроспективное наблюдение 45 малазийских пациентов с ОАК показало, что 11 больных $(24,4 \%)$ после в/с введения ГиК больше не нуждались в дальнейшем лечении ОА [22].

Кроме того, препараты ГиК имеют низкую частоту НЯ, сопоставимую с плацебо (чаще всего быстро проходящие местные реакции - боль, отек и артралгии), поэтому они могут быть альтернативой обезболивающим препаратам, особенно у пожилых больных с высокой коморбидностью, и, более того, дают возможность отсрочить необходимость в эндопротезировании (ЭПС) коленных суставов от 2 до 3,6 года [23-26].
Препараты ГиК, также как и другие SYSADOA, обладают хондропротективным (снижение апоптоза и увеличение пролиферации хондроцитов, подавление экспрессии ММП-13, увеличение синтеза протеогликанов/гликозаминогликанов), анальгетическим и противовоспалительным (уменьшение активности ИЛ-1 $\beta$, ИЛ-6 и др.) действием [27].

В литературе имеется большое количество исследований, продемонстрировавших эффективность и безопасность в/с инъекций ГиК при лечении ОА разных локализаций.

Препарат Гиалон (Fidia Farmaceutici S.p.A.) представляет собой стерильный апирогенный вязкоупругий раствор, изготовленный из натриевой соли ГиК, полученной путем бактериальной ферментации с высоким молекулярным весом в диапазоне 1500-2000 кДа, который широко используется в лечении ОА. Это новое линейное, т. е. несшитое химическое производное ГиК, демонстрирующее уникальные реологические свойства, сходные с нативной ГиК в синовиальной жидкости. Препарат Гиалон (60 мг/4 мл) вводится в сустав однократно, курс проводят 1-2 раза в год. Для трехкратного введения, а также после артроскопии применяется препарат Хиалубрикс (30 мг/2 мл) [28].

В нескольких наблюдательных и контролируемых клинических исследованиях доказана эффективность и безопасность препарата. Так, в многоцентровом крупномасштабном наблюдательном исследовании 1523 пациента (81,3\% с ОАК) получили три внутрисуставных инъекции Хиалубрикс и наблюдались в течение шести недель. Интенсивность боли и ФН оценивались по визуальной аналоговой шкале (ВАШ) как в начале, так и в конце лечения. 91,2\% пациентов сообщили о значительном уменьшении боли на фоне терапии, а $85,3 \%$ - о значительном улучшении или полном восстановлении подвижности сустава по сравнению с тем, что было до начала лечения [29].

В работе C. Фоти (C. Foti) и соавт. приняли участие 1266 пациентов из 47 центров с ОА различной локализации (коленный сустав $-82,0 \%$ от общего числа суставов; тазобедренный сустав - 9,0\%, плечевой - 6,3\%; предплюсне-предплюсневый сустав $-1,5 \%$; запястно-пястный сустав - $1,2 \%)$. Препарат вводился в/с 1 раз в неделю в течение 3 недель в соответствующей размеру сустава дозе. Результаты показали статистически значимое улучшение по сравнению с исходным визитом при оценке боли, ФН и качества жизни. Частота НЯ составила 0,8\% (95\% ДИ от 0,4 до 1,5). Сообщалось о 13 НЯ, 12 из которых были легкой или средней степени тяжести. Серьезных НЯ отмечено не было [30].

В многоцентровом, рандомизированном, открытом контролируемом исследовании, в которое было включено 100 пациентов, Ф. Приано (F. Priano) и соавт. установили, что больные, получавшие ГиК после менискэктомии $(\mathrm{N}=51)$, испытывали значительно меньшую боль при ходьбе, в состоянии покоя, во время активных движений и имели лучшую подвижность суставов, чем пациенты, не получавшие инъекций ГиК $(\mathrm{N}=49)$ [31].

Дж. Филардо (G. Filardo) и соавт. сравнили инъекцию плазмы, обогащенной тромбоцитами (PRP), с инъекцией препарата Хиалубрикс 30 мг для лечения ОАК. При всех контрольных визитах (через 2, 6 и 12 месяцев после лечения) исследователи отметили, что 3 еженедельные инъекции ГиК (2 мл/30 мг каждая, $\mathrm{N}=89$ ) были так же эффективны, как и 3 еженедельные инъекции PRP по 5 мл ( $=94)$ [32]. Данные исследования показали, что Хиалубрикс имеет хороший профиль безопасности, НЯ в основном имели незначительные клинические проявления (покраснение, зуд или боль в месте инъекции). 
Частота НЯ в крупных исследованиях варьировала от 0,5\% до $0,8 \%[29,30]$.

Внутрисуставное введение ГиК в тазобедренные суставы стало более востребовано при использовании ультразвукового (УЗ) контроля, так как УЗ-наведение улучшает и повышает точность доставки препарата, помогает при повторных инъекциях, что очень важно при длительном лечении ОА тазобедренного сустава. Так, в открытом исследовании А. Мильоре (A. Migliore) и соавт. 1906 пациентов (4002 инъекции), которым вводили ГиК под УЗ-контролем, подтвердили, что данные инъекции эффективны, хорошо переносятся с небольшими и исключительно местными НЯ, что позволяет проводить длительное лечение ОА [33, 34].

Далее А. Мильоре и соавт. изучили эффективность однократного в/с введения препарата Гиалон (4 мл/60 мг) у 344 пациентов с ОА тазобедренных суставов 1-3 стадии по Келлгрену и Лоуренсу. Препарат вводился каждые 6, а при необходимости - каждые 3 месяца под УЗ-контролем. Длительность исследования составила 18 месяцев. Результаты показали значительное снижение как индекса Лекена и боли по ВАШ, так и потребности в НПВП во всех временных точках по сравнению с исходным уровнем. К концу 18 -месячного периода наблюдения только $3 \%$ пациентов подверглись хирургическому лечению (ЭПС тазобедренного сустава) [35].

В 2011 г. эти результаты были подтверждены в проспективном когортном исследовании с участием пациентов с симптоматическим ОА тазобедренных суставов I, II, III или IV стадий по Келлгрену-Лоуренсу в возрасте $\geqslant 40$ лет, испытывающих боль в течение как минимум одного года. В работе приняли участие 120 пациентов, которым вводили Гиалон 4 мл/60 мг в/с каждые 6 месяцев, а при необходимости - каждые 3 месяца под У3-контролем, причем наблюдение длилось тоже в течение 18 месяцев. Статистически значимое снижение альгофункциональных показателей отмечалось через 3 месяца после первой инъекции. Через 12 месяцев у $80 \%$ пациентов симптомы уменьшились более чем на $30 \%$. Достигнутые результаты сохранялись с течением времени на фоне повторных инъекций, выполняемых не реже одного раза в 6 месяцев. НЯ были незначительными и встречались примерно в $3 \%$ случаев [36].

Такие наблюдения свидетельствуют о том, что повторные в/с инъекции Гиалона у пациентов с ОА тазобедренных суставов сохраняют длительный анальгетический эффект и возможность снизить прием НПВП, что особенно важно у пожилых пациентов с сопутствующими заболеваниями.

Терапевтическая эффективность препарата также была подтверждена в двойном слепом контролируемом исследовании по сравнению с в/с введением анестетика мепивакаина гидрохлорида у пациентов с ОА тазобедренных суставов. Основная цель исследования заключалась в сравнении альгофункционального индекса Лекена в двух группах через 6 месяцев наблюдения после первой инъекции. Вторичные цели заключались в сравнении двух групп по интенсивности боли, потребности в НПВП и общей субъективной и объективной глобальной оценке состояния. Было включено 42 пациента, 85,7\% из которых имели ОА 3-й стадии. Через 3 и 6 месяцев наблюдения отмечалось значимое снижение индекса Лекена в обеих группах по сравнению с исходным уровнем, однако препарат ГиК значительно превосходил мепивакаин в обеих временных точках $-7,09 \pm 3,78$ и 5,15 \pm 5,15 и $3,94 \pm 2,58$ против $7,75 \pm 4,15$ и $6,53 \pm 4,33$ и $6,41 \pm 4,14$ соответственно. Боль тоже значительно уменьшилась по сравнению с исходным уровнем и была гораздо слабее в основной группе $-6,4 \pm 1,94$ и 4,3 $\pm 2,58$ и 4,5 $\pm 1,96$ против $6,0 \pm 1,34$

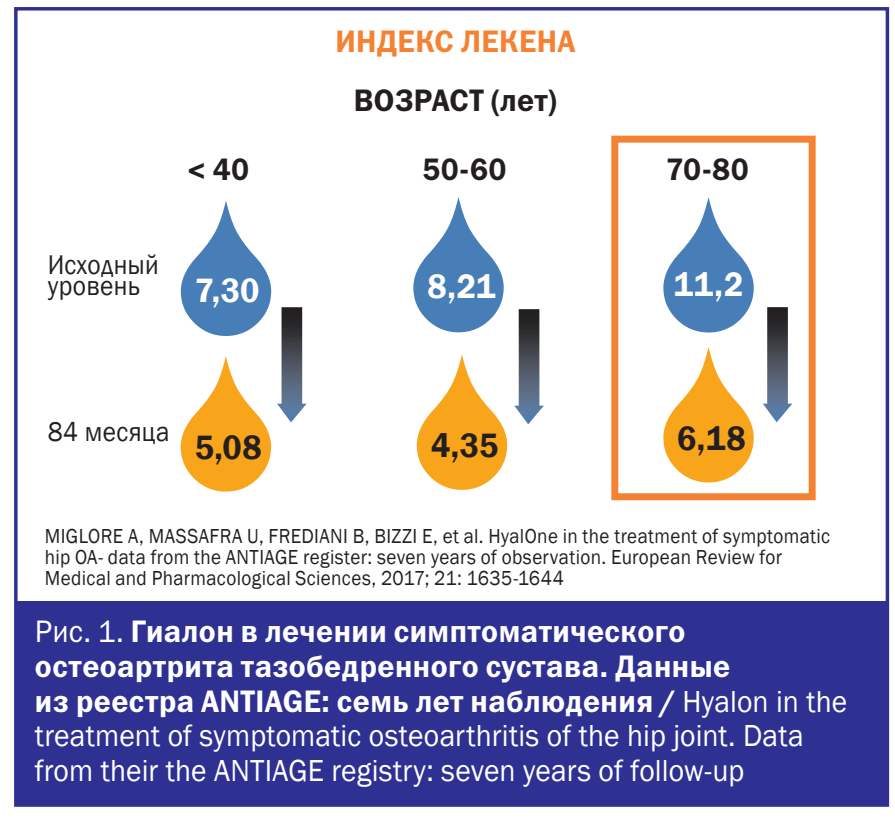

и 4,5 $\pm 2,63$ и 5,0 $\pm 2,41$ соответственно. У всех пациентов снизилась потребность в НПВП, а также в обеих группах отмечалось улучшение субъективной и объективной глобальных оценок состояния, но без статистически значимых различий. Серьезных НЯ отмечено не было [37].

Для оценки влияния терапии на замедление прогрессирования заболевания и/или возможности задержки замены сустава авторами было проведено дополнительное исследование длительностью 48 месяцев. Шесть независимых ортопедов оценивали 176 пациентов с ОА тазобедренных суставов кандидатов на ЭПС, получавших внутрисуставные инъекции ГиК под контролем УЗИ каждые 6 месяцев с дополнительными инъекциями каждые 3 месяца при необходимости. 159 из 176 (90\%) через 24 месяца и 144 (82\%) пациента через 48 месяцев не подверглись ЭПС. Таким образом, было показано, что регулярные в/с инъекции препарата Гиалон могут отсрочить проведение ЭПС у больных симптоматическим ОА тазобедренных суставов [38].

В 2017 г. были опубликованы результаты длительного 7-летнего исследования, собранные из реестра Национальной ассоциации внутрисуставной терапии тазобедренного сустава с ультразвуковым контролем (ANTIAGE) по оценке эффективности в/с инъекций препарата Гиалон (60 мг/4 мл), повторяемых не менее 2 раз в год у большой когорты пациентов с ОА тазобедренных суставов под контролем УЗИ. Значения индекса Лекена, боли по ВАШ, потребность в НПВП оценивались каждые 3 месяца до конца периода наблюдения. Рентгенологическое обследование проводилось через каждые 24 месяца. 1022 включенных пациента были распределены по возрасту, полу и индексу массы тела (ИМТ). Гиалон продемонстрировал эффективность во всех возрастных группах, в том числе у пожилых пациентов (рис. 1). Показатели боли значимо сильнее уменьшились $(46,7 \%)$ у пациентов от 40 лет и моложе. У больных старше 70 лет также наблюдалось соответствующее ослабление боли с постепенным уменьшением до $25 \%$. Пациенты из группы старше 80 лет продемонстрировали улучшение функциональных показателей примерно в 45\% случаев при последнем контрольном посещении, при оценке с другими возрастными категориями - от $34 \%$ до $50 \%$. При избыточном весе и ожирении различий по полу не было. 
Тем не менее у женщин отмечались более высокий исходный уровень боли и потребность в НПВП по сравнению с мужчинами, у которых через 84 месяца уровень индекса Лекена оказался более низким. Авторы предположили, что наблюдаемые различия способны помочь в выявлении больных, лучше отвечающих на лечение препаратами ГиК.

Полученные результаты свидетельствуют о том, что все пациенты, независимо от возраста или ИМТ, отметили значительное улучшение по всем оцениваемым показателям через 6 месяцев после лечения, и, что наиболее важно, на фоне повторных инъекций ГиК такое улучшение сохранялось в течение последующих 6,5 лет. За 7 лет ни разу не сообщалось о системных или тяжелых местных НЯ. Некоторые пациенты после инъекции испытывали боль, которая длилась от нескольких часов до нескольких дней. Таким образом, исследование показало, что Гиалон, демонстрируя отличный профиль безопасности, не терял эффективности после повторных инъекций в течение многих лет [39].

Вместе с тем внутрисуставное введение препаратов, в том числе ГиК, должно проводиться по строгим показаниям и в специальных условиях, поскольку безопасность любого метода является одним из главных принципов проводимого лечения. B 2020 г. на конгрессе EULAR были представлены рекомендации по внутрисуставному введению препаратов, созданные экспертами из 8 стран (рис. 2). Использование этих рекомендаций позволит стандартизировать данную процедуру, что будет способствовать повышению эффективности и лучшей безопасности этого уже признанного метода лечения.

Таким образом, результаты проведенных исследований демонстрируют высокую симтоматическую эффективность

\footnotetext{
- Пациент Аолжен быть хорошо информирован о природе вмешательства, потенциальных рисках и пользе, Аолжно быть подписано информированное согласие

- Оптимальные условия Аля внутрисуставного лечения:

- Специально поАготовленный, чистый, индивиАуальный, хорошо освещенный кабинет

- Условия Аля асептического проведения процеАуры

- Meacectpa

- Набор аля экстренной помощи

- Точность введения зависит от сустава, места введения и профессиональной подготовки врача, при возможности необходимо использовать УЗ-навигацию

- Во время беременности необходимо уточнить, безопасен ки вводимый препарат Аля матери и плоАа

- ВсегАа Аолжна использоваться асептическая техника введения

- Пациенту необходимо объяснить плюсы и минусы локальной анестезии
}

- Пациенты с Аиабетом, особенно при недостаточно хорошо контролируемом, Аолжны быть преАупреждены о риске транзиторной гипергликемии после внутрисуставного ввеАения ГиК, о необхоАимости Аополнительного контроля уровня глюкозы в течение трех Аней после инъекции

- Внутрисуставное введение не противопоказано пациентам с нарушением свертывания крови или получающим антиагрегантную терапию, кроме случаев с высоким риском кровотечения

- Внутрисуставное введение может проводиться более чем за 3 месяца Ао планируемого эндопротезирования

- Решение о повторном внутрисуставном введении нужно принимать с учетом пользы от преАыдущих ввеАений и инАивидуальных факторов (варианты лечения, препараты, системное лечение, сопутствующие заболевания)

- Избегать повышенных нагрузок на сустав в течение 24 часов после инъекции, иммобилизация не рекомендуется

Рис. 2. Рекомендации EULAR по внутрисуставному лечению артропатий / EULAR recommendations for intraarticular arthropathy treatment препарата Гиалон при ОАК и тазобедренных суставов: значимое уменьшение боли, улучшение функции суставов, снижение потребности в НПВП, что в свою очередь приводит к уменьшению НЯ, вызванных приемом препаратов данной группы, что очень важно у мультиморбидных больных ОА. Препарат обладает высокой безопасностью и может применяться в разных возрастных группах. Повышение мобильности пациентов приводит к улучшению качества жизни и, как следствие, может снизить немедикаментозные затраты, связанные с оказанием им помощи. Такая терапия может длиться годами, не теряя своей пользы и не вызывая значимых НЯ. Кроме того, Гиалон обладает болезнь-модифицирующим действием, поскольку отдаляет необходимость проведения ЭПС.

КОНФЛИКТ ИНТЕРЕСОВ. АвторЫ статьИ ПоАТверАИ^и отсУтствие КоНфликта интересов, о котором необходимо сообщить.

CONFLICT OF INTERESTS. Not declared.

\section{ヘитература/References}

1. Алексеева Л. И., Таскина Е. А., Кашеварова Н. Г.

Остеоартрит: эпидемиология, классификация, факторы риска и прогрессирования, клиника, диагностика, лечение // Современная ревматология. 2019; 13 (2): 9-21.

[Alekseeva L. I., Taskina E. A., Kashevarova N. G.

Osteoartrit: epidemiologiya, klassifikatsiya, faktory riska I proressirovaniya, klinika, diagnostika, lechenie [Osteoarthritis: epidemiology, classification, risk factors, and progression, clinical presentation, diagnosis, and treatment] // Modern Rheumatology Journal. 2019; 13 (2): 9-21. (In Russ.)] Available from: https://doi.org/10.14412/19967012-2019-2-9-21.

2. Cross M., Smith E., Hoy D., et al. The global burden of hip and knee osteoarthritis: estimates from the global burden of disease 2010 study // Ann Rheum Dis. 2014; 73 (7): 1323-1330.

DOI: 10.1136/annrheumdis-2013-204763.

3. Nüesch E., Dieppe P., Reichenbach $S$. et al. All cause and disease specific mortality in patients with knee or hip osteoarthritis: population based cohort study // BMJ. 2011; 342: d1165. DOI: 10.1136/bmj.d1165.

4. Bannuru R. R., Osani M. C., Vaysbrot E. E. et al. OARSI guidelines for the non-surgical management of knee, hip, and polyarticular osteoarthritis // Osteoarthritis Cartilage. 2019; 27 (11): 1578-1589. DOI: 10.1016/j.joca.2019.06.011.

5. Bruyère O., Honvo G., Veronese N. et al. An updated algorithm recommendation for the management of knee osteoarthritis from the European Society for Clinical and Economic Aspects of Osteoporosis, Osteoarthritis and Musculoskeletal Diseases (ESCEO). Semin Arthritis Rheum // Semin Arthritis Rheum. 2019; pii: S0049-0172 (19): 30043-30045. DOI: 10.1016/j. semarthrit.2019.04.008.

6. Kolasinski L. Sharon, Neogi T., Hochberg C. Marc. et al. 2019 American College of Rheumatology/Arthritis Foundation Guideline for the Management of Osteoarthritis of the Hand, Hip, and Knee // Arthritis Care \& Research. 2020: 1-14. DOI: $10.1002 /$ acr.24131.

7. Kloppenburg M., Kroon Féline P. B., Blanco J. Francisco et al. // Ann Rheum Dis. 2019; 78: 16-24. DOI: 10.1136/annrheumdis-2018-213826.

8. Министерство здравоохранения Российской Федерации. Остеоартроз. Клинические рекомендации. М., 2016. [Ministry of Health of the Russian Federation. Osteoarthritis. Clinical recommendations. M., 2016.] 
9. Georgiev T., Angelov A. K. Modifiable risk factors in knee osteoarthritis: treatment implications // Rheumatol Int. 2019; 39 (7): 1145-1157. DOI: 10.1007/s00296-019-04290-z.

10. Meyer K., Palmer J. The polysaccharide of the vitreous humor // J. Biol. Chem. 1934; 107: 629-634.

11. Necas J., Bartosikova L., Brauner P., Kolar J. Hyaluronic acid (hyaluronan): A review // Vet. Med. 2008; 53: 397-411. DOI: 10.17221/1930-VETMED.

12. Kogan G., Šolté L., Stern R., Mendichi R. Hyaluronic acid: A biopolymer with versatile physico-chemical and biological properties. In: Pethrick R. A., Ballada A., Zaikov G. E., editors. Handbook of Polymer Research: Monomers, Oligomers, Polymers and Composites. Nova Science Publishers; Hauppauge, NY, USA: 2007. P. 393-439.

13. Fallacara A., Baldini E., Manfredini S., Vertuani S. Hyaluronic Acid in the Third Millennium // Polymers (Basel). 2018; 10 (7).

14. De Oliveira J. D., Carvalho L. S., Gomes A. M., Queiroz L. R., Magalhães B. S., Parachin N. S. Genetic basis for hyper production of hyaluronic acid in natural and engineered microorganisms // Microb Cell Fact. 2016; 15 (1): 119.

15. Wang Y., Hu L., Huang H., Wang H., Zhang T., Chen J., Du G., Kang $Z$. Eliminating the capsule-like layer to promote glucose uptake for hyaluronan production by engineered Corynebacterium glutamicum // Nat Commun. 2020; 11 (1): 3120.

16. Boeriu C. G., Springer J., Kooy F. K., van den Broek L. A. M., Eggink $G$. Production methods for hyaluronan // Int. J. Carbohydr. Chem. 2013; 2013: 1-14. DOI: $10.1155 / 2013 / 624967$.

17. Laurent T. C., Laurent U. B., Fraser J. R. The structure and function of hyaluronan: An overview // Immunol Cell Biol. 1996; 74 (2): A1-7.

18. Burmester G., Lanas A., Biasucci L., Hermann M., Lohmander S., Olivieri I., Scarpignato C., Smolen J., Hawkey C., Bajkowski A., Berenbaum F., Breedveld F., Dieleman P., Dougados M., MacDonald T., Mola E. M., Mets T., van den Noortgate N., Stoevelaar H. The appropriate use of non-steroidal anti-inflammatory drugs in rheumatic disease: opinions of a multidisciplinary European expert panel // Ann Rheum Dis. 2011; 70 (5): 818-822.

19. Bannuru R. R., Natov N. S., Obadan I. E., Price L. L., Schmid C. H., McAlindon T. E. Therapeutic trajectory of hyaluronic acid versus corticosteroids in the treatment of knee osteoarthritis: a systematic review and meta-analysis // Arthritis Rheum. 2009; 61: 1704-1711. DOI: 10.1002/art.24925.

20. Narayanan S., Suhail A., Harjeet S., Shahril Y., Masbah O. Efficacy of intra-articular injection of hyaluronic acid in the treatment of knee osteoarthritis // Malaysian Orthop J. 2009; 3 (1): 19-23. DOI: 10.5704/MOJ.0905.002.

21. Bannuru R. R., Schmid C. H., Kent D. M., Vaysbrot E. E., Wong J. B., McAlindon T. E. Comparative effectiveness of pharmacologic interventions for knee osteoarthritis: a systematic review and network meta-analysis // Ann Intern Med. 2015; 162 (1): 46-54.

22. Richette P., Chevalier X., Ea H. K., Eymard F., Henrotin Y., Ornetti $P$., et al. Hyaluronan for knee osteoarthritis: an updated meta-analysis of trials with low risk of bias // Rheum Musculoskelet Dis. 2015; 1: e000071.

23. Delbarre A., Amor B., Bardoulat I., Tetafort A., PelletierFleury $N$. Do intra-articular hyaluronic acid injections delay total knee replacement in patients with osteoarthritis A Cox model analysis // PLoS One. 2017; 12 (11): e0187227.

24. Cooper C., Rannou O. I. S., Richette P., Ere O. B., Al-daghri N., Altman R. O. Y. D., et al. Use of Intraarticular hyaluronic acid in the Management of Knee Osteoarthritis in clinical practice // Arthritis Ann Intern Med Care Res. 2017; 69 (9): 1287-1296. DOI: 10.1002/acr.23204.

25. Altman R., Lim S., Steen R. G., Dasa V. Hyaluronic acid injections are associated with delay of Total knee replacement surgery in patients with knee osteoarthritis: evidence from a large U.S. health claims database // PLoS One. 2015; 10 (12): e0145776. DOI: 10.1371/journal.pone.0145776.

26. Altman R., Fredericson M., Bhattacharyya S. K., Bisson B., Abbott T., Yadalam M. B. A. S., et al. Association between hyaluronic acid injections and time-to-Total knee replacement surgery // J Knee Surg. 2016; 29 (7): 564-570. DOI: $10.1055 / \mathrm{s}-0035-1568992$.

27. Altman R. D., Manjoo A., Fierlinger A., Niazi F., Nicholls M. The mechanism of action for hyaluronic acid treatment in the osteoarthritic knee: a systematic review // BMC Musculoskelet Disord. 2015; 16: 321. DOI: 10.1186/s12891-015-0775-z.

28. Finelli I., Chiessi E., Galesso D., Renier D., Paradossi G. A new viscosupplement based on partially hydrophobic hyaluronic acid: a comparative study // Biorheology. 2011; 48 (5): 263-75.

29. Schieb F. Intraartikulärinjizierte-Hyaluronsäurebeiarthropathien // Arthritis Rheum. 2003; 6: 338-340.

30. Foti C., Cisari C., Carda S., Giordan N., Rocco A., Frizziero A., Della Bella $G$. A prospective observational study of the clinical efficacy and safety of intra-articular sodium hyaluronate in synovial joints with osteoarthritis // Eur J Phys Rehabil Med. 2011; 47 (3): 407-415.

31. Priano F., Guelfi M. Efficacy of intra-articular hyaluronic acid (Hyalubrix $^{\circledR}$ ) in arthroscopy // Artroscopia. 2007; 8: 1-12.

32. Filardo G., Di Matteo B., Di Martino A., et al. Platelet-Rich Plasma Intra-articular Knee Injections Show No Superiority Versus Viscosupplementation: A Randomized Controlled Tria // Am J Sports Med. 2015; 43 (7): 1575-1582.

33. Migliore A., Martin L. S., Alimonti A., et al. Efficacy and safety of viscosupplementation by ultrasound-guided intraarticular injection in osteoarthritis of the hip // Osteoarthritis Cartilage. 2003; 11 (4): 305-306.

34. Migliore A., Tormenta S., Laganà B., et al. Safety of intra-articular hip injection of hyaluronic acid products by ultrasound guidance: an open study from ANTIAGE register // Eur Rev Med Pharmacol Sci. 2013; 17 (13): 1752-1759.

35. Migliore A., Bizzi E., Massafra U., et al. 18 months followup after intra-articular administration of Hyalubrix ${ }^{\circledR}$ in 344 patients with symptomatic osteoarthritis of the hip // Osteoarthritisand Cartilage. 2008; 16 (S4): S118-S119.

36. Migliore A., Massafra U., Bizzi E., et al. Intra-articular injection of hyaluronic acid (MW 1,500-2,000 kDa; HyalOne) in symptomatic osteoarthritis of the hip: a prospective cohort study // Arch Orthop Trauma Surg. 2011; 131 (12): 1677-1685.

37. Migliore A., Massafra U., Bizzi E., et al. Comparative, doubleblind, controlled study of intra-articular hyaluronic acid (Hyalubrix) injections versus local anesthetic in osteoarthritis of the hip // Arthritis Res Ther. 2009; 11 (6): R183.

38. Migliore A., Bella A., Bisignani M., et al. Total hip replacement rate in a cohort of patients affected by symptomatic hip osteoarthritis following intra-articular sodium hyaluronate (MW 1,500-2,000 kDa) ORTOBRIX study // Clin Rheumatol. 2012; 31 (8): 1187-1196.

39. Migliore A., Massafra U., Frediani B., et al. HyalOne ${ }^{\circledR}$ in the treatment of symptomatic hip OA - data from the ANTIAGE register: seven years of observation // Eur Rev Med Pharmacol Sci. 2017; 21 (7): 1635-1644. 